The Astrophysical Journal, 470:L123-L126, 1996 October 20

(c) 1996. The American Astronomical Society. All rights reserved. Printed in U.S.A.

\title{
ACCRETION DISKS AROUND CLASS 0 PROTOSTARS: THE CASE OF VLA 1623
}

\author{
Ralph E. Pudritz, ${ }^{1}$ Christine D. Wilson, ${ }^{1}$ John E. Carlstrom, ${ }^{2}$ Oliver P. Lay, ${ }^{3}$ Richard E. Hills, ${ }^{4}$ and Derek Ward-Thompson ${ }^{5}$ \\ Received 1996 May 28; accepted 1996 August 5
}

\begin{abstract}
Continuum emission at 220 and $355 \mathrm{GHz}$ from the prototype class 0 source VLA 1623 has been detected using the James Clerk Maxwell Telescope-Caltech Submillimeter Observatory interferometer. Gaussian fits to the data place an upper limit of $70 \mathrm{AU}$ on the half-width at half-maximum radius of the emission, which implies an upper limit of $\sim 175 \mathrm{AU}$ for the cutoff radius of the circumstellar disk in the system. In the context of existing collapse models, this disk could be magnetically supported on the largest scales and have an age of $\sim 6 \times 10^{4} \mathrm{yr}$, consistent with previous suggestions that class 0 sources are quite young. The innermost region of the disk within $\sim 6 \mathrm{AU}$ is likely to be in centrifugal support, which is likely large enough to provide a drive for the outflow according to current theoretical models. Alternatively, if 175 AU corresponds to the centrifugal radius of the disk, the age of the system is $\sim 2 \times 10^{5} \mathrm{yr}$, closer to age estimates for class I sources.

Subject heading: accretion, accretion disks - circumstellar matter — stars: formation —

stars: low-mass, brown dwarfs — stars: pre-main sequence
\end{abstract}

\section{INTRODUCTION}

Submillimeter observations of deeply embedded star-forming regions have identified a small number of strong sources that have virtually no emission at wavelengths below $10 \mu \mathrm{m}$ and whose spectral energy distributions are characterized by single blackbodies at $T \simeq 15-30 \mathrm{~K}$. These cold "class 0 " sources are good candidates for protostars whose circumstellar envelope masses exceed that of their central protostars (André, Ward-Thompson, \& Barsony 1993; André \& Montmerle 1994; André 1995), in which case their ages may be as low as $2 \times 10^{4}$ yr (Barsony 1994). Sources in this class are also defined by the presence of energetic bipolar outflows.

There is now good evidence that a large fraction of young stellar objects have dense, dusty accretion disks (e.g., Beckwith et al. 1990; Lada 1991). This is in accord with standard collapse models of star formation (e.g., Terebey, Shu, \& Cassen 1984), which predict that systems older than $10^{5} \mathrm{yr}$ have centrifugally supported disks extending to 100 AU. Disks around the sources L1551 IRS 5 and HL Tau have been resolved with semimajor axes of 80 and 60 AU, respectively (Lay et al. 1994; Mundy et al. 1996). Even larger magnetically supported "pseudodisks" extending out to 1000 AU are predicted for some magnetohydrodynamic collapse models (Galli \& Shu 1993; Fiedler \& Mouschovias 1993).

Most current models of outflow posit the existence of disks as an essential element of the engine (e.g., Pudritz, Pelletier, \& Gomez de Castro 1991; Königl \& Ruden 1993; Shu et al. 1993). If the class 0 sources are young, then the Terebey et al. (1984) models suggest that a disk with a radius of $100 \mathrm{AU}$ at $2 \times 10^{5} \mathrm{yr}$ has a centrifugal radius of only $r_{c} \propto t^{3} \simeq 0.8 \mathrm{AU}$ at $2 \times 10^{4}$ yr. Similarly, a larger, magnetically supported outer region of $r_{B} \propto t^{7 / 3} \simeq 20 \mathrm{AU}$ (Galli \& Shu 1993) should be present at these early times if 1000 AU structures exist at

${ }^{1}$ Department of Physics and Astronomy, McMaster University, Hamilton, Ontario L8S 4M1, Canada.

2 Department of Astronomy and Astrophysics, University of Chicago, 5640 South Ellis Avenue, Chicago, IL 60637.

${ }^{3}$ Division of Physics, Mathematics, and Astronomy, 103-33, California Institute of Technology, Pasadena CA 91125.

${ }^{4}$ Mullard Radio Astronomy Observatory, Madingley Road, Cambridge CB3 0HE, England, UK.

${ }^{5}$ Royal Observatory, Blackford Hill, Edinburgh, EH9 3HJ, Scotland, UK.
$2 \times 10^{5}$ yr. Such small, centrifugally supported regions would still be able to drive outflows, while the larger magnetic structure could play an important role in star formation itself. This Letter describes the results of our search for an accretion disk in the prototype class 0 source, VLA 1623, using the James Clerk Maxwell Telescope-Caltech Submillimeter Observatory (JCMT-CSO) interferometer.

\section{OBSERVATIONS AND ANALYSIS}

Observations of VLA 1623 were obtained with the JCMTCSO interferometer on 1995 April 7 at a frequency of $356 \mathrm{GHz}$ with $500 \mathrm{MHz}$ bandwidth and on 1995 April 9 at $353 \mathrm{GHz}$ with $1 \mathrm{GHz}$ bandwidth. The projected length of the baseline ranged from 140 to $190 \mathrm{k} \lambda$, and observations were obtained over a period of 2-3 hr each night. The quasar NRAO 530 was used to monitor the gain of the instrument, and the absolute uncertainty in the flux calibration is $20 \%$. The average gain of the instrument was $70 \mathrm{Jy} \mathrm{K}^{-1}$, and the system temperature was $800 \mathrm{~K}$. The data were obtained with $10 \mathrm{~s}$ integrations and vector-averaged over $100 \mathrm{~s}$ intervals. Since the phase response of the interferometer over longer periods of time is not yet known, only the amplitude of the visibility function was used in the analysis. Observations were also obtained at a frequency of $220 \mathrm{GHz}$ with $1 \mathrm{GHz}$ bandwidth on 1995 April 10 over a period of $7 \mathrm{hr}$. The projected length of the baseline ranged from 60 to $120 \mathrm{k} \lambda$, and the system temperature was $400 \mathrm{~K}$.

The visibility flux of VLA 1623 shows no dependence on the projected baseline length (Fig. 1, top panels), and thus the source is unresolved. We measured both the flux and an upper limit to the FWHM radius of the source by using the techniques outlined in Lay et al. (1994). Briefly, we fitted a two-dimensional Gaussian to the data by using the Rice distribution to describe the noise. The position angle was fixed at $35^{\circ}$ to orient the disk perpendicular to the large-scale outflow (André et al. 1990). Fifty simulated data sets were then generated by adding artificial noise to the best-fitting model. The best-fit parameters (flux and major-axis radius) derived from these simulated data sets are shown in Figure 1 (bottom right panel), from which $90 \%$ confidence limits can be determined. At $355 \mathrm{GHz}$, the flux is $0.5_{-0.1}^{+0.4} \mathrm{Jy}$, and the 

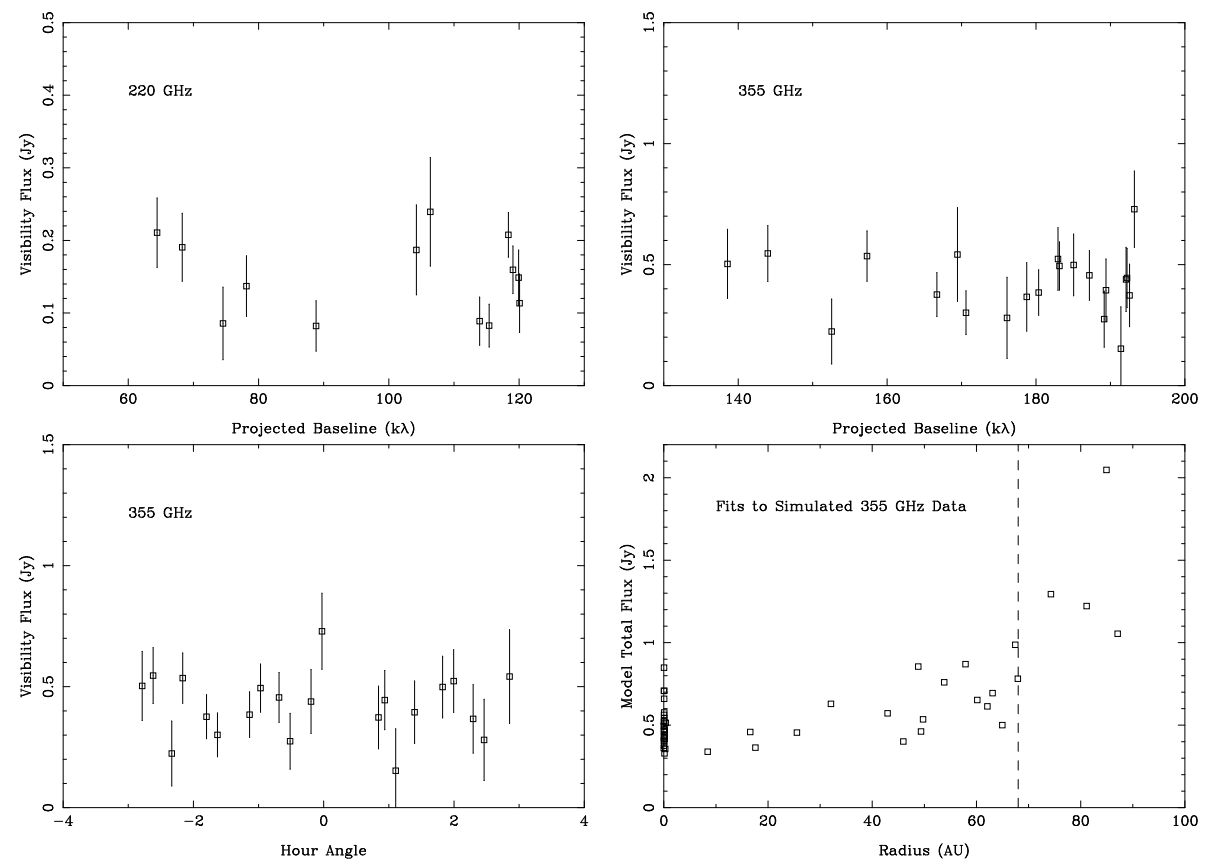

FIG. 1.-(Top left panel): the visibility flux of VLA 1623 at $220 \mathrm{GHz}$ detected with the JCMT-CSO interferometer as a function of the projected baseline length. The flux shows no dependence on projected baseline length, and thus the source is unresolved. (Top right panel): the visibility flux at $355 \mathrm{GHz}$ as a function of baseline length. (Bottom left panel) The visibility flux at $355 \mathrm{GHz}$ as a function of the hour angle of the observations. (Bottom right panel): the source flux at $355 \mathrm{GHz}$ deduced from 50 Monte Carlo simulations as a function of the deduced upper limit to the source size. With the available data, it is impossible to distinguish between a weak compact source and a more extended, somewhat stronger source. The dashed line indicates the $90 \%$ confidence level upper limit to the radius of the disk.

major-axis radius is $R<70 \mathrm{AU}$ (for a distance to $\rho \mathrm{Oph}$ of $160 \mathrm{pc})$. Fitting the data with a circularly symmetric Gaussian produces a somewhat smaller upper limit to the radius of 45 AU. A more physically valid model would be a power-law disk (see $\S 3$ ). The large uncertainty in the flux at $355 \mathrm{GHz}$ is due to the rather limited baseline coverage obtained for this low-declination source. As a result, the model fits are unable to distinguish between a weaker compact source and a stronger, more extended source for which some of the flux is resolved out even on the shortest baselines at $355 \mathrm{GHz}$ (Fig. 1). At $220 \mathrm{GHz}$, the flux is $0.12_{-0.02}^{+0.08} \mathrm{Jy}$, and the radius is $R<$ 150 AU.

We calculated the expected contribution to the visibility flux from the extended envelope as in Lay et al. (1994). We modeled the envelope of VLA 1623 as an optically thin sphere with $T \propto r^{-0.5}$ and $\rho \propto r^{-1.5}$. The flux scale was calibrated using single-dish fluxes from André et al. (1993). We also assumed that the envelope stops where the disk begins, and so we adopted an inner cutoff radius for the envelope of $70 \mathrm{AU}$. Under these conditions, the envelope for the $70 \mathrm{AU}$ model contributes negligible flux at $355 \mathrm{GHz}$ and only $0.03 \mathrm{Jy}$ at $220 \mathrm{GHz}$. Thus, we attribute most of the observed flux to the presence of an unresolved disk in VLA 1623.

\section{FITTING ACCRETION DISK MODELS}

Our observations provide useful constraints on plausible accretion disk models for the origin of the submillimeter emission. We adopt the approach of Beckwith et al. (1990) and assume a geometrically thin disk with power-law scalings with radius of the disk temperature and surface density, $T(r)=$ $T_{0}\left(r / r_{0}\right)^{-q}$ and $\Sigma(r)=\Sigma_{0}\left(r / r_{0}\right)^{-p}$, where $T_{0}$ and $\Sigma_{0}$ are the dust temperature and mass surface density at the inner radius $r_{0}$ of the dust distribution. The optical depth is $\tau=\Sigma \kappa_{v} / \cos \theta$, where $\theta$ is the inclination of the disk axis to the line of sight and $\kappa_{\nu}=\kappa_{0}\left(\nu / \nu_{0}\right)^{\beta}$ is the specific dust opacity. The flux emitted by the disk is then $F_{v}=\left(2 \pi \cos \theta / D^{2}\right) \int_{r_{0}}^{R_{D}} B_{v}(T)\left(1-e^{-\tau}\right) r d r$, where $B_{v}(T)$ is the Planck function, $D$ is the distance to the source, and $R_{D}$ is the outer disk cutoff radius. Power-law fits to disks around HL Tau and L1551 IRS5 produce cutoff radii up to a factor of 2.5 larger than radii from Gaussian fits (Lay et al. 1994; Mundy et al. 1996). However, since VLA 1623 is unresolved, there is no structure in the visibility data to constrain a power law, and so such a fit was not performed on this data set.

While $q=0.75$ for purely viscous accretion disk models, more general calculations that incorporate the effects of reprocessing and disk flaring typically find $0.5 \leq q \leq 0.75$. Noting that various model fits have $0.5 \leq p \leq 1.5$ (e.g., Thamm, Steinacker, \& Henning 1994), we adopt ranges of $0.25 \leq q \leq 0.75$ and $-0.25 \leq 2-p-q \leq 1.25$. The maximum dust temperature must be less than its sublimation temperature, $T_{0, \max } \sim 2000 \mathrm{~K}$. The inner edge of the disk cannot be inside the stellar photosphere, which for low-mass stars gives $r_{0} \geq R_{*} \simeq 2 R_{\odot}=0.01 \mathrm{AU}$. We take the inclination angle of the disk to be that of the outflow from VLA 1623, $\theta=80^{\circ} \pm 5^{\circ}$ or almost edge-on (André et al. 1990). The spectrum $F_{\nu} \propto \nu^{\alpha}$ determined from our measured fluxes at 355 and $220 \mathrm{GHz}$ has $\alpha \simeq 3.0_{-2.2}^{+1.6}$, where optically thin models have $\alpha=2+\beta$ and optically thick models have $\alpha=2$. Recent calculations show that $\beta \simeq 1.5 \pm 0.5$ at wavelengths from $650 \mu \mathrm{m}$ to $2.7 \mathrm{~mm}$ for submicron to millimeter-sized particles and a wide variety of densities and temperatures (Pollack et al. 1994). Finally, the parameter space characterizing the emission is also constrained by our observed flux, our upper limit to the cutoff radius of $\sim 2.5 \times 70=175 \mathrm{AU}$, and by the $\sim 1 L_{\odot}$ bolometric luminosity of the entire source (André et al. 1993).

It is obvious that this accretion disk model covers a large parameter space that is relatively poorly constrained. The issue of the uniqueness of model fits has been addressed by 

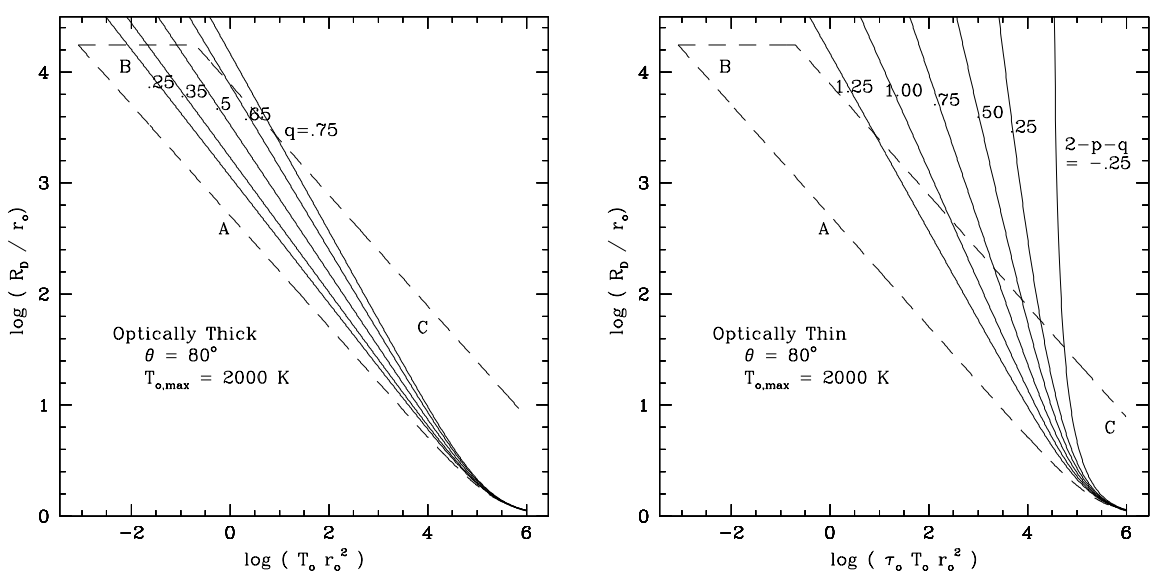

FIG. 2.-(Left panel): cutoff radius $R_{D}$ of an optically thick disk in units of the inner disk radius $r_{0}$ vs. the product $T_{0} r_{0}^{2}$, where $T_{0}$ is the temperature of the disk at radius $r_{0}$. The solid lines are model fits for different values of $q$, the exponent in the temperature gradient. Viable accretion disk models lie within the region demarcated by the dashed-line segments, A, B, and C, described in the text. The angle between the disk axis and the line of sight is $\theta=80^{\circ}$. (Right panel): same as in the left panel, but for optically thin emission. In this case, $R_{D}$ depends on the product $\tau_{0} T_{0} r_{0}^{2}$.

Thamm et al. (1994) and Bouvier \& Bertout (1992), who find "global ambiguity" in acceptable fits to models of T Tauri disks based on spectral energy distributions that incorporate far more infrared data than is available for VLA 1623. However, for the limiting cases of completely optically thick or thin truncated disks in the Rayleigh-Jeans limit, the cutoff radius can be related to the observed flux $F_{\nu}$ by

$$
R_{D} / r_{0}=\left[\left(\gamma \bar{L}_{v} / x \cos \theta\right)+1\right]^{1 / \gamma},
$$

where $\bar{L}_{\nu}=D^{2} F_{v} / 2 \pi\left(2 k \nu^{2} / c^{2}\right)$ can be computed from the observations. For the optically thick case, the parameters are $\gamma=$ $2-q$ and $x=T_{0} r_{0}^{2}$, while for the optically thin case, $\gamma=2-$ $p-q \neq 0$ and $x=\tau_{0} T_{0} r_{0}^{2}$, where $\tau_{0} \equiv \kappa_{\nu} \Sigma_{0} / \cos \theta$.

Equation (1) shows that we can simplify our analysis of the model by reducing the six free parameters $\left(\kappa_{\nu}, q, p, T_{0}, r_{0}\right.$, and $\Sigma_{0}$ ) to essentially the two parameters $\gamma$ and $x$ (with $R_{D}$ measured in units of $r_{0}$ ). Then a disk model characterized by a given index $\gamma$ produces a unique curve in the $R_{D} / r_{0}-x$ plane. These curves are plotted in Figure 2 for VLA 1623, for which $F_{v}=0.5 \mathrm{Jy}$ gives $\bar{L}_{355}=2.25 \times 10^{4} \mathrm{~K} \mathrm{AU}^{2}$. In addition, our observed flux and upper limit to the cutoff radius restrict possible disk models to lie within the region demarcated by the three dashed lines in Figure 2. Boundary line A is the $\gamma=2$ model (i.e., $q=0$ or $p+q=0$ for optically thick or thin cases), which demarcates a model disk with uniform surface brightness and therefore the smallest cutoff radius $R_{D}$ for the emitted flux. Line B is our upper limit to the cutoff radius $R_{D} \leq 175 \mathrm{AU}$ coupled with the condition that $r_{0} \geq 0.01 \mathrm{AU}$, while line $\mathrm{C}$ is the upper limit to $R_{D} / r_{0}$ for a given $x$. Line $\mathrm{C}$ arises from the fact that $T_{0}$ must be less than $T_{0, \max }$, so that $r_{0}$ must be greater than $\left(x / T_{0, \max }\right)^{1 / 2}$, and thus $R_{D} \leq 175 \mathrm{AU}$ implies $R_{D} / r_{0} \leq 175\left(T_{0, \max } / x\right)^{1 / 2}$. Figure 2 shows that, while optically thick models with values of $q \simeq 0.25-0.5$ fill the largest portion of the permitted parameter space, models with $q$ up to 0.75 are also acceptable. Optically thin models fill a much smaller portion of parameter space, with low values of $2-p-q$ being less likely. In general, the optically thin solutions have relatively large values for the inner radius, which suggests that they may also have a small, optically thick region that contributes relatively little to the total flux of the source.

The observed bolometric luminosity of the envelope + disk system also provides a constraint on the cutoff radius. For $q \neq$
0.5 , the luminosity of an optically thick disk is given by $L_{\mathrm{bol}}=$ $4 \pi \sigma T_{0}^{4} r_{0}^{2}\left[\left(R_{D} / r_{0}\right)^{2-4 q}-1\right] /(2-4 q)$, while for $q=0.5$, $L_{\mathrm{bol}}=4 \pi \sigma T_{0}^{4} r_{0}^{2} \ln \left(R_{D} / r_{0}\right)$. For $q<0.5$, this expression can be combined with equation (1) to give

$$
\begin{aligned}
R_{D}= & 47.7 \mathrm{AU} \frac{(2-q)^{2 / 3}}{(2-4 q)^{1 / 6}}\left(\frac{L_{\mathrm{bol}}}{1 L_{\odot}}\right)^{-1 / 6} \\
& \times\left(\frac{L_{355} / 2.25 \times 10^{4} \mathrm{~K} \mathrm{AU}^{2}}{\cos \theta / \cos 80}\right)^{2 / 3} .
\end{aligned}
$$

In addition, equation (1) can be rewritten as $T_{0} r_{0}^{q} \propto R_{D}^{q-2}$, which allows the straightforward calculation of $T_{0} r_{0}^{q}$ for a given $R_{D}$ obtained from equation (2). For $q=0$ and $L_{\mathrm{bol}}<1$ $L_{\odot}$, the cutoff radius is $R_{D}>68 \mathrm{AU}$ and the inner disk temperature is $T_{0}<57 \mathrm{~K}$, while for $q=0.25$ and $r_{0}=$ 0.01-10 AU, the radius is greater than $69 \mathrm{AU}$ and the temperature is $T_{0}<430-80 \mathrm{~K}$. For $q \geq 0.5$, the expression for $R_{D}$ is different from equation (2) and depends on the value of $r_{0}$. For $q=0.5$ and $r_{0}=0.01-1 \mathrm{AU}$, the cutoff radius is greater than $90-85 \mathrm{AU}$, while for $q=0.75$, the radius is greater than 230-120 AU. A smaller, but warmer, optically thin disk could probably also produce the observed $355 \mathrm{GHz}$ flux and bolometric luminosity, but the numerical models required to derive the exact cutoff radius in this case are beyond the scope of this Letter.

Thus, both the spectral index and the models summarized in Figure 2 are consistent with either optically thin or optically thick disks with a cutoff radius not exceeding 175 AU. We obtain a lower limit to the disk mass from the measured $220 \mathrm{GHz}$ flux by taking the optically thin limit,

$$
M \geq\left[D^{2} F_{\nu} / \kappa_{\nu} B_{\nu}\left(T_{0}\right)\right](2-p-q / 2-p)\left(R_{D} / r_{0}\right)^{q} .
$$

Assuming a cutoff radius $R_{D}=175 \mathrm{AU}$, an inner radius $r_{0}=$ $0.01 \mathrm{AU}$, an opacity $\kappa_{v}=0.01 \mathrm{~cm}^{2} \mathrm{~g}^{-1}$ at $220 \mathrm{GHz}$ $(\beta=1.5)$, a maximum temperature of $T_{0, \max }=430 \mathrm{~K}$, and $q \simeq 0.25$ gives a minimum disk mass $M \geq 3 \times 10^{-2} M_{\odot}$; the true disk mass could be much higher.

\section{DISCUSSION}

The constraints on the size of the central source have implications for theoretical models of the evolution of sources such as VLA 1623. We adopt an effective sound speed $a=$ 
$0.27 \mathrm{~km} \mathrm{~s}^{-1}$ for this innermost region of VLA 1623 (André et al. 1993) and a magnetic field strength $B=30 \mu \mathrm{G}$ appropriate for low-mass molecular cloud cores (Myers \& Goodman 1988). In the context of the Galli \& Shu (1993) model, the magnetic support radius of the disk is given by

$$
\begin{aligned}
r_{B} & =0.12\left(G^{2} B^{4} / a\right)^{1 / 3} t^{7 / 3} \\
& =2.75(B / 30 \mu \mathrm{G})^{4 / 3}\left(a / 0.27 \mathrm{~km} \mathrm{~s}^{-1}\right)^{-1 / 3} t_{4}^{7 / 3} \mathrm{AU},
\end{aligned}
$$

where $t_{4}$ is in units of $10^{4} \mathrm{yr}$ and $G$ is the gravitational constant. If we identify the upper limit to the cutoff radius of $175 \mathrm{AU}$ with the radius of the pseudodisk, $r_{B}$, then this model predicts an age for the system of $t<5.9 \times 10^{4}(B / 30 \mu \mathrm{G})^{-4 / 7}\left(a / 0.27 \mathrm{~km} \mathrm{~s}^{-1}\right)^{1 / 7} \mathrm{yr}$. This age agrees with general estimates for class 0 ages (Barsony 1994) but is somewhat longer than initial estimates for the age of VLA 1623 (André et al. 1993). In a collapsing singular isothermal sphere (SIS) model, the expansion wave has reached a radius $r_{\text {exp }}=a t_{\text {exp }}<3400\left(t_{\exp } / 5.9 \times 10^{4} \mathrm{yr}\right) \mathrm{AU}$, which is comparable to the size of the 2000 AU envelope seen in the André et al. (1993) map. In the context of the SIS model, this age suggests that the envelope and the collapsed protostellar core + disk will each contain comparable amounts of material.

Alternatively, if we identify $175 \mathrm{AU}$ with the centrifugal balance radius, $r_{c}=0.058 a \Omega_{0}^{2} t^{3}=0.030\left(\Omega_{0} / 10^{-13} \mathrm{~s}^{-1}\right)^{2} t_{4}^{3}$ $\mathrm{AU}$, then the age of the system is less than $1.8 \times 10^{5} \mathrm{yr}$, similar to the age estimates for class I sources (Wilking, Lada, \& Young 1989). In this picture, the magnetic pseudodisk, if present, would extend to $2300 \mathrm{AU}$ and must have a very low column density in order to be undetectable with lower resolution measurements (e.g., André et al. 1993). This age is 5 times longer than the time required for the expansion wave to reach the edge of the circumstellar envelope. Over this time, a compressional wave propagating inward from the envelope edge would have accelerated the collapse of the remaining envelope material onto the stellar core.

Returning to the assumption that most of the compact core is magnetically supported, the centrifugal balance radius in the pseudodisk then occurs at $r_{c}<6.2\left(\Omega_{0} / 10^{-13} \mathrm{~s}^{-1}\right)^{2}$ AU. This region is large enough either to drive off a disk wind or to interact with a possible stellar magnetosphere, and thus theoretical models developed to explain outflows in class I sources are likely to be equally valid for class 0 sources. The total collapsed mass of the star + disk system in the SIS model is $M_{\text {coll }} \simeq[1+(\pi / 4)]^{-1} 2 a^{2} r_{\text {exp }} / G=0.29 M_{\odot}$. Taking a stellar radius $R_{*}=2.5 R_{\odot}$ (valid for models with $M_{*}<0.3 M_{\odot}$; Stahler 1988) and a bolometric luminosity $L_{*} \sim 1 L_{\odot}$ (André et al. 1993), we find that an accretion rate through the disk of $\dot{M}_{a}=L_{*} R_{*} / G M_{*}=0.27 \times 10^{-6} M_{\odot} \mathrm{yr}^{-1}$ can provide the observed luminosity of VLA 1623. This accretion rate is significantly smaller than the rate inferred from the collapse of an SIS model, $\dot{M}_{i}=0.975 a^{3} / G=4.5 \times 10^{-6} M_{\odot} \mathrm{yr}^{-1}$. A discrepancy of a factor of 5 between the disk and the collapse accretion rates in SIS models has been noted by Kenyon, Calvet, \& Hartmann (1993) for more evolved T Tauri systems. A possible reason for the inconsistency in the mass accretion rates is that SIS models have constant infall rates. More general models of molecular cloud cores (e.g., McLaughlin \& Pudritz 1996, 1997; Foster \& Chevalier 1993) do not collapse at a constant accretion rate, which may alleviate this problem.

Finally, we note that the outflow in VLA 1623 has reached much larger scales ( 20,000 AU; André et al. 1990) than the expansion wave predicted by the SIS model. This situation is possible because outflows are likely to be super-Alfvénic. The effect that such an outflow has upon collapse has yet to be investigated.

\section{CONCLUSIONS}

High-resolution submillimeter continuum observations have detected a compact source in VLA 1623, which is most readily interpreted as a disk. Gaussian fitting gives an upper limit of 70 $\mathrm{AU}$ for the half-width at half-maximum radius, which implies an upper limit of $\sim 175$ AU for the cutoff radius of the circumstellar disk in the system. In the context of existing collapse models, such a structure could be magnetically supported on the largest scales and have an age $\sim 6 \times 10^{4} \mathrm{yr}$. The innermost region of the disk within $\sim 6 \mathrm{AU}$ is likely to be in centrifugal support, which could provide a drive for the outflow according to current theoretical models. Alternatively, if the entire observed disk is in centrifugal support, then the age of the system is $\sim 2 \times 10^{5} \mathrm{yr}$, similar to the age estimates of class I sources. In this case, we may be detecting a class I source viewed nearly edge-on.

We thank the referee, Lee Mundy, in particular, for pointing our attention to the limit provided by the bolometric luminosity of the source. C. D. W. and R. E. P. thank Peter Sutherland for help with numerical fitting techniques. The research of R. E. P. and C. D. W. is supported through grants from the Natural Sciences and Engineering Research Council of Canada. J. E. C. gratefully acknowledges support from a NSF-YI Award, and a David and Lucile Packard Fellowship. The JCMT is operated by the Royal Observatories on behalf of the Particle Physics and Astronomy Research Council of the United Kingdom, the Netherlands Organization for Scientific Research, and the National Research Council of Canada. Research at the CSO is supported by NSF grant 90-15755.

\section{REFERENCES}

André, P. 1995, Ap\&SS, 224, 29

André, P., Martin-Pintado, J., Despois, D., \& Montmerle, T. 1990, A\&A, 236, 180

André, P., \& Montmerle, T. 1994, ApJ, 420, 837

André, P., Ward-Thompson, D., \& Barsony, M. 1993, ApJ, 406, 122

Barsony, M. 1994, in ASP Conf. Ser. 65, Clouds, Cores, and Low Mass Stars, ed. D. Clemens \& R. Barvainis (San Francisco: ASP), 197

Beckwith, S. V. W., Sargent, A. I., Chini, R. S., \& Güsten, R. 1990, AJ, 99, 924 Bouvier, J., \& Bertout, C. 1992, A\&A, 263, 113

Fiedler, R. A., \& Mouschovias, T., Ch. 1993, ApJ, 415, 680

Foster, P. N. \& Chevalier, R. A. 1993, ApJ, 416, 303

Galli, D., \& Shu, F. H. 1993, ApJ, 417, 243

Kenyon, S. J., Calvet, N., \& Hartmann, L. 1993, ApJ, 414, 676

Königl, A., \& Ruden, S. P. 1993, in Protostars and Planets III, ed. E. H. Levy \& J. I. Lunine (Tucson: Univ. Arizona Press), 641

Lada, C. J. 1991, in The Physics of Star Formation and Early Stellar Evolution, ed. C. J. Lada \& N. D. Kylafis (Dordrecht: Kluwer), 329
Lay, O. P., Carlstrom, J. E., Hills, R. E., \& Phillips, T. G. 1994, ApJ, 434, L75 McLaughlin, D. E., \& Pudritz, R. E. 1996, ApJ, 469, 194

. 1997, ApJ, submitted

Mundy, L. G., et al. 1996, ApJ, 464, L169

Myers, P. C., \& Goodman, A. A. 1988, ApJ, 326, L31

Pollack, J. B., Hollenbach, D., Beckwith, S., Simonelli, D. P., Roush, T., \& Fong, W. 1994, ApJ, 421, 615

Pudritz, R. E., Pelletier, G., \& Gomez de Castro, A. I. 1991, in The Physics of

Star Formation and Early Stellar Evolution, ed. C. J. Lada \& N. D. Kylafis (Dordrecht: Kluwer), 539

Shu, F., Najita, J., Galli, D., Ostriker, E., \& Lizano, S. 1993, in Protostars and Planets III, ed. E. H. Levy \& J. I. Lunine (Tucson: Univ. Arizona Press), 3 Stahler, S. W. 1988, ApJ, 332, 804

Terebey, S., Shu, F. H., \& Cassen, P. 1984, ApJ, 286, 529

Thamm, E., Steinacker, J., \& Henning, Th. 1994, A\&A, 287, 493

Wilking, B. A., Lada, C. J., \& Young, E. T. 1989, ApJ, 340, 823 